\title{
The diagnosis of a non-stenotic bicuspid aortic valve
}

\author{
GRAHAM LEECH, PETER MILLS, AND AUBREY LEATHAM
}

From The Cardiac Department, St George's Hospital, Hyde Park Corner, London

SUMMARY Follow-up of a group of subjects in whom an aortic ejection sound was the only abnormal finding revealed a bicuspid aortic valve at necropsy or operation in 6 cases. High speed echophonocardiographic studies in 15 subjects with aortic stenosis and known to have bicuspid valves, showed the ejection sound to be exactly synchronous with final halting of the opening aortic vaive cusps. Echocardiographic visualisation of the aortic valve from the apex, looking up the left ventricular outflow tract, showed valve echoes during systole indicating abnormal cusp configuration. This proved a more sensitive indicator of a bicuspid aortic valve than the finding of an eccentric aortic valve closure line.

These findings were used to evaluate 37 subjects with the auscultatory finding of an isolated aortic ejection sound and the diagnosis of a non-stenotic bicuspid aortic valve was confirmed in 30 . The usual cause of misdiagnosis was a sound associated with late tricuspid valve closure.

The auscultatory finding of an aortic ejection sound, in the absence of other signs of aortic stenosis, indicates a non-stenotic, bicuspid aortic valve. Confirmation of aortic valvar origin of the sound can be provided by a simultaneous echophonocardiogram.

The observation that an early systolic sound might be associated with an abnormal aortic valve was first noted by Lian and Welti (1937), and in 1940 Wolferth and Margolies suggested that this sound could be regarded as an 'opening click' of a diseased aortic valve. Leatham and Vogelpoel (1954) described it as an 'aortic ejection sound', and showed that it occurred approximately at the time of the carotid upstroke. Cineangiographic studies by Epstein et al. (1965) subsequently disclosed that in aortic valve stenosis the ejection sound coincided with the halting of the upward 'doming' movement of the fused aortic valve cusps. It is now generally accepted that in patients with obstruction to left ventricular outflow, an ejection sound indicates the presence of aortic valve stenosis with valve cusps that are reasonably mobile and not heavily calcified (Hancock, 1961; Oakley and Hallidie-Smith, 1967; Glancy and Epstein, 1971).

It has, however, been noted that an early systolic sound may occur in patients in whom there is no detectable degree of aortic stenosis (Leatham et al., 1963). In view of the observation that a non-stenotic bicuspid aortic valve is a common congenital anomaly of the heart (Wauchope, 1928),we suggested that this might be the origin of the early systolic sound in these patients.

Received for publication 19 October 1977
The present study was designed to examine this hypothesis and to develop criteria for the noninvasive recognition of a bicuspid aortic valve. Combined echo- and phonocardiography offers a non-invasive method for studying the motion of cardiac valves and their associated sounds. This can be used, in a manner analogous to the cineangiographic techniques of Epstein et al. (1965), to identify the aortic valve as the origin of a suspected aortic ejection sound.

\section{Subjects and methods}

\section{PATIENTS}

All the patients in this study had an early systolic sound approximately synchronous with the beginning of the external carotid pulse upstroke. None had evidence of any abnormality apart from the aortic valve. No patient had systemic hypertension or rheumatic heart disease.

Group 1 consisted of 6 patients in whom an isolated aortic ejection sound, documented phonocardiographically, was subsequently shown to have been associated with a bicuspid aortic valve. In 4 patients, a non-stenotic bicuspid aortic valve was found at necropsy. The other 2 patients developed aortic stenosis, and at operation a calcified, congenitally bicuspid aortic valve was found. 
Group 2: The echocardiographic fealures of a bicuspid aortic valve and the timing relation between valve motion and the associated ejection sound were studied in 15 patients with aortic stenosis. Of these, 10 had undergone surgery; in each case the valve had been found to be bicuspid, but its anatomy was not altered by the procedure, which was either a limited valvotomy or debridement. In the remaining 5 , the diagnosis of a bicuspid aortic valve had been established unequivocally by angiography.

Group 3 comprised 37 patients referred for cardiac assessment, in whom an early systolic sound, thought to be an ejection sound, was noted on auscultation. There was no evidence of aortic stenosis and the chest $x$-ray film and electrocardiogram were normal.

As controls 30 subjects without any evidence of cardiac abnormality were studied.

\section{METHODS}

A commercial echoscope ${ }^{1}$ with $2 \cdot 25 \mathrm{MHz}$ focused transducer was modified to increase the pulse repetition rate from 1000 to $2000 / \mathrm{s}$. The output was coupled via a shift register interface to a 6-channel oscilloscopic strip chart recorder, ${ }^{2}$ allowing simultaneous recording of $\mathrm{M}$-mode echo, two phonos, electrocardiogram, external carotid pulse, and respiration. The recorder resolution was improved by doubling the echo depth magnification and increasing the paper speed to $200 \mathrm{~mm} / \mathrm{s}$. This system is capable of resolving tissue depths to $0.5 \mathrm{~mm}$ and time intervals to $\pm 2.5 \mathrm{~ms}$. Registration delay between channels (including echo) is negligible (less than $0.3 \mathrm{~ms}$ ).

The following studies were performed on all patients, except 5 of the 6 in group 1 who were seen before echocardiography was available.

(1) A routine echocardiogram.

(2) High speed echophonocardiograms (Leatham and Leech, 1975) to define the relation between mitral and tricuspid valve closure, aortic and pulmonary valve opening, and the heart sounds recorded at the pulmonary area and left sternal border. Recordings of each valve were made after the patient had achieved a steady resting state and a regular heart rate.

(3) The aortic valve was also visualised, with the transducer placed over the cardiac apex. The ultrasound beam was directed medially and cephalad towards the mid-point of the right clavicle, to locate the mitral valve. From this point, the aortic valve was located by angling still more medially

${ }^{1}$ Smith Kline Instrument Co. Ekoline 20.

${ }^{2}$ Cambridge Medical Instruments Model 72112. and cephalad. The appearance of the aortic valve in this view is similar to that of the pulmonary valve from the third left intercostal space. These recordings will be referred to as apical aortic valve echocardiograms.

Standard high frequency phonocardiograms were recorded from two chest sites simultaneously (pulmonary area and apex, followed by aortic area and left sternal border), together with the indirect carotid pulse using a small funnel pickup, respiration phase, and electrocardiogram. From these recordings, the following measurements were made

(a) The time interval from the onset of the high frequency vibrations of the first heart sound to the onset of the ejection sound.

(b) The relative amplitudes of the first sound, ejection sound, and second sound at the apex, in all phases of the respiratory cycle.

(c) The relation between the ejection sound and the onset of the external carotid pulse upstroke.

(d) Carotid pulse tracings were used to measure the 'T-time', that is time to half maximal upstroke excursion (Epstein and Coulshed, 1964), and left ventricular ejection time (Weissler et al., 1968).

\section{Results}

The measurements are summarised in the Table and are discussed below.

\section{GROUP 1}

Phonocardiograms had previously been recorded on the 6 patients subsequently shown at necropsy or surgery to have a bicuspid aortic valve. Fig. 1 illustrates the features of the early systolic sound seen in these patients. It is a high frequency sound, occurring 45 to 62 (mean 55) ms after the onset of the first heart sound, and shortly before the upstroke of the indirect carotid pulse. Recordings at the apex showed that the sound was usually as loud as the first heart sound and its intensity did not vary with respiration. The aortic component of the second sound was louder than normal, averaging 3.5 times the amplitude of the first sound, compared with a ratio of 2.0 in the controls. In the only case in whom $N$ echocardiographic studies were performed, the sound started exactly at the moment of halting of the opening aortic valve cusps. Five of the six had a $\omega$ systolic murmur graded from 1 to $2 / 6$, but none

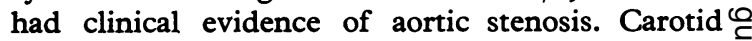
'T-time' and left ventricular ejection time indices were within normal limits in each patient.

\section{GROUP 2}

Each of the 15 patients in group 2, known to have a stenotic bicuspid aortic valve, had an ejection 
Table Summary of results

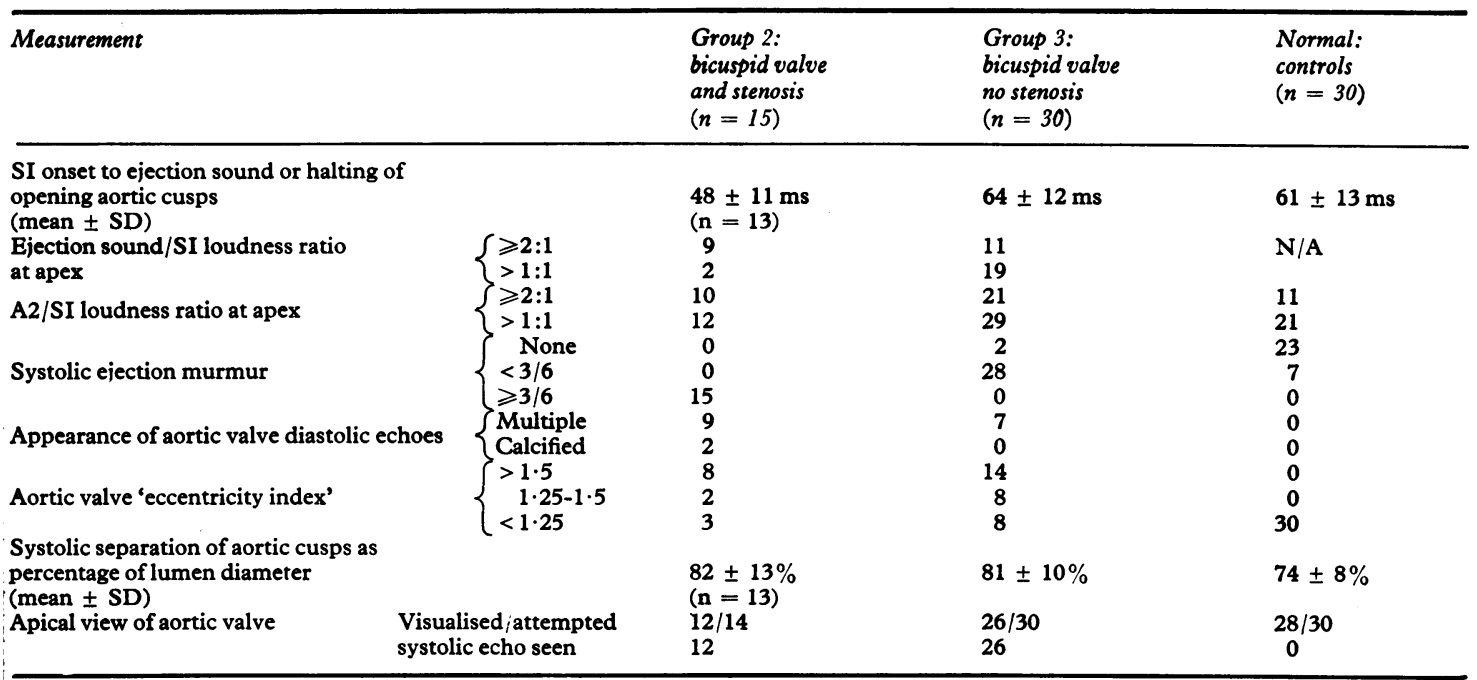

sound 30 to 65 (mean 48) ms after the onset of the first sound. Echophonocardiographic studies showed that the onset of the ejection sound coincided with halting of the opening movement of the aortic valve cusps in the conventional view and in the apical view (Fig. 2). In each patient, both the mitral and tricuspid valves had closed, and the pulmonary valve had opened, before maximal aortic valve opening. Evaluation of the aortic valve echoes in diastole was not possible in two patients, who had multiple dense echoes in the aortic root suggesting the presence of calcium and precluding further measurements. Of the remaining 13 patients with proven bicuspid valves, the 'eccentricity index' of
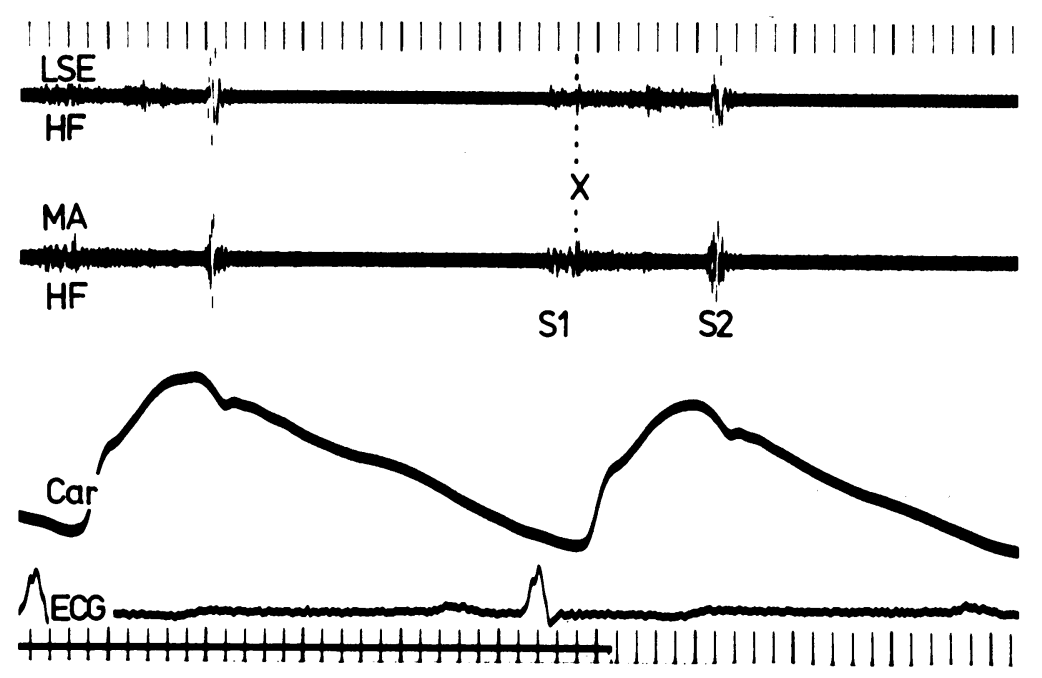

Fig. 1 Phonocardiogram showing high frequency sound tracings recorded at left sternal edge ( $L S E)$ and mitral area $(M A)$, with carotid pulse (Car) and electrocardiogram (ECG), recorded in 1962. The patient had an early systolic sound $(X)$, thought from its relation to the carotid pulse upstroke to be an aortic ejection sound. He subsequently died of myocardial infarction and was shown at necropsy to have a non-stenotic bicuspid aortic valve. $H F$, high frequency; $S 1$, first sound;

$S 2$, second sound. 
Fig. 2 Selection of recordings from a patient in group 2 with a surgically proven bicuspid valve. Top left: phonocardiogram showing ejection sound $(X)$ and systolic murmur $(S M)$; the carotid pulse upstroke is slow. Lower left: echo recording of tricuspid valve leaflets (TVL), showing closure (TVC) at apposition of anterior $(a)$ and posterior $(p)$ leaflets, occurring well before the ejection sound. Top right: conventional aortic valve echo showing closed leaflets $(A V L)$ anteriorly displaced within the aortic lumen $(A 0)$. Onset of the ejection sound vibrations coincides precisely with halting of the opening of the valve (AVO). Lower right: apical view, showing valve echoes during systole and relationship of opening to ejection sound. All recordings at $200 \mathrm{~mm} / \mathrm{s}$. Time lines $40 \mathrm{~ms}$.

the aortic valve echo during diastole (Nanda et al., 1974) was greater than 1.5 in 8 patients and greater than 1.25 in 2 more. In the other 3 patients, however, the eccentricity index was below 1.25 and the overall appearance of the valve from the conventional transducer position was normal. Visualisation of the aortic valve from the apex was successful in 12 cases. In each instance, the aortic cusp echoes were recorded during most of systole, suggesting that part of the cusp structure remained in the central portion of the lumen of the aorta during ejection (Fig. 2 lower right panel), an appearance never found in the control group.

\section{GROUP 3}

Thirty-seven patients thought on auscultation to have an aortic ejection sound were studied using the same techniques. In 30 of these patients, the onset of the sound coincided with maximal aortic valve opening, confirming the clinical impression of an aortic ejection sound (Fig. 3). The interval from the onset of the first heart sound to the ejection $\bigcirc$ sound and maximal aortic valve opening was 43 to 91 (mean 64) ms. This was essentially the same as 은 the interval from the first sound onset to maximal $>$ aortic opening in the normal control group, and highly significantly $(P<0.001)$ longer than in the N group 2 patients with aortic stenosis. In 19/30 the ejection sound was louder than the first sound at 0 the apex, and in the majority, the aortic component $\omega$ of the second sound was accentuated. A soft? systolic murmur was present in 28 , and a very softco early diastolic murmur in 4 cases. Carotid pulse $\Phi$ 'T-time' and left ventricular ejection time indices? were normal (except in one case of unoperated $\underline{T}$ coarctation of the aorta) and there was no clinical $\frac{O}{\mathbb{D}}$ evidence of aortic stenosis.

The aortic valve eccentricity index exceeded $1.5 \stackrel{\vec{Q}}{\stackrel{Q}{Q}}$ 


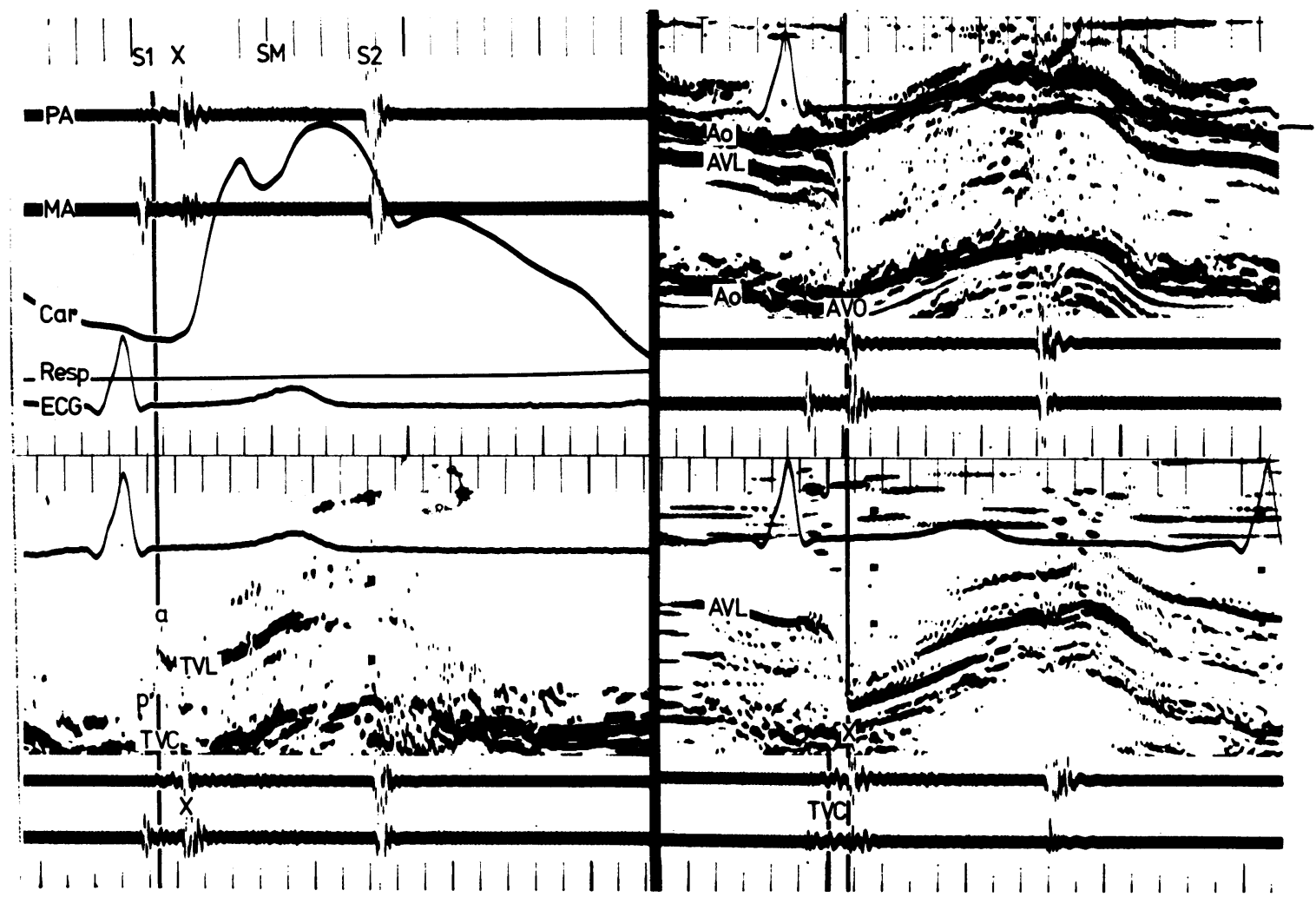

Fig. 3 Recordings from a patient in group 3 arranged in the same way as Fig. 2. Note the similarity, apart from the minimal murmur and normal carotid rise time, suggesting no stenosis.

in 14 cases, was between 1.5 and 1.25 in 8 , but was less than 1.25 in 8 of the 30 cases. Multiple diastolic echoes were found in 7 patients. In none was there evidence of aortic valve calcification. The initial separation of the aortic cusp echoes at the beginning of ejection, expressed as a percentage of the aortic lumen diameter, was significantly greater $(P<0.01)$ in groups 2 and 3 than in the normal controls. The aortic valve was successfully visualised from the apex in 26 of these 30 cases and in every instance valve echoes could be seen during systole.

In the remaining 7 patients in group 3 , the sound thought on auscultation to be an aortic ejection sound did not coincide with maximal aortic valve opening. In 5, it coincided with tricuspid valve closure and preceded maximal aortic opening (Fig. 4). Moreover, its intensity increased on inspiration and it was best heard at the lower left sternal border. In one of these, the tricuspid component was very late and loud and the electrocardiogram was compatible with a mild form of Ebstein's anomaly; in another, with a moderately loud systolic murmur, there were echocardiographic signs suggestive of discrete subvalvar aortic obstruction; the other 3 had relatively widely split first heart sounds (up to $40 \mathrm{~ms}$ ), but without any electrocardiographic evidence of conduction delay. In the remaining 2 of the 7 patients, the ejection sound was shown to coincide with maximal pulmonary valve opening, its intensity diminished on inspiration, and the pulmonary component of the second heart sound was delayed, suggesting mild pulmonary valve stenosis. In none of these patients was the aortic valve echo seen during systole in the apical view, nor were eccentric or multiple diastolic echoes seen in the conventional aortic valve echocardiogram.

\section{CONTROLS}

None of the 30 patients without evidence of cardiac disease had a sound coinciding with maximal aortic valve opening. Echocardiographically, the eccentricity index of aortic valve closure was always less than $1 \cdot 25$ and the aortic cusps were never recorded during systole in the apical view. 


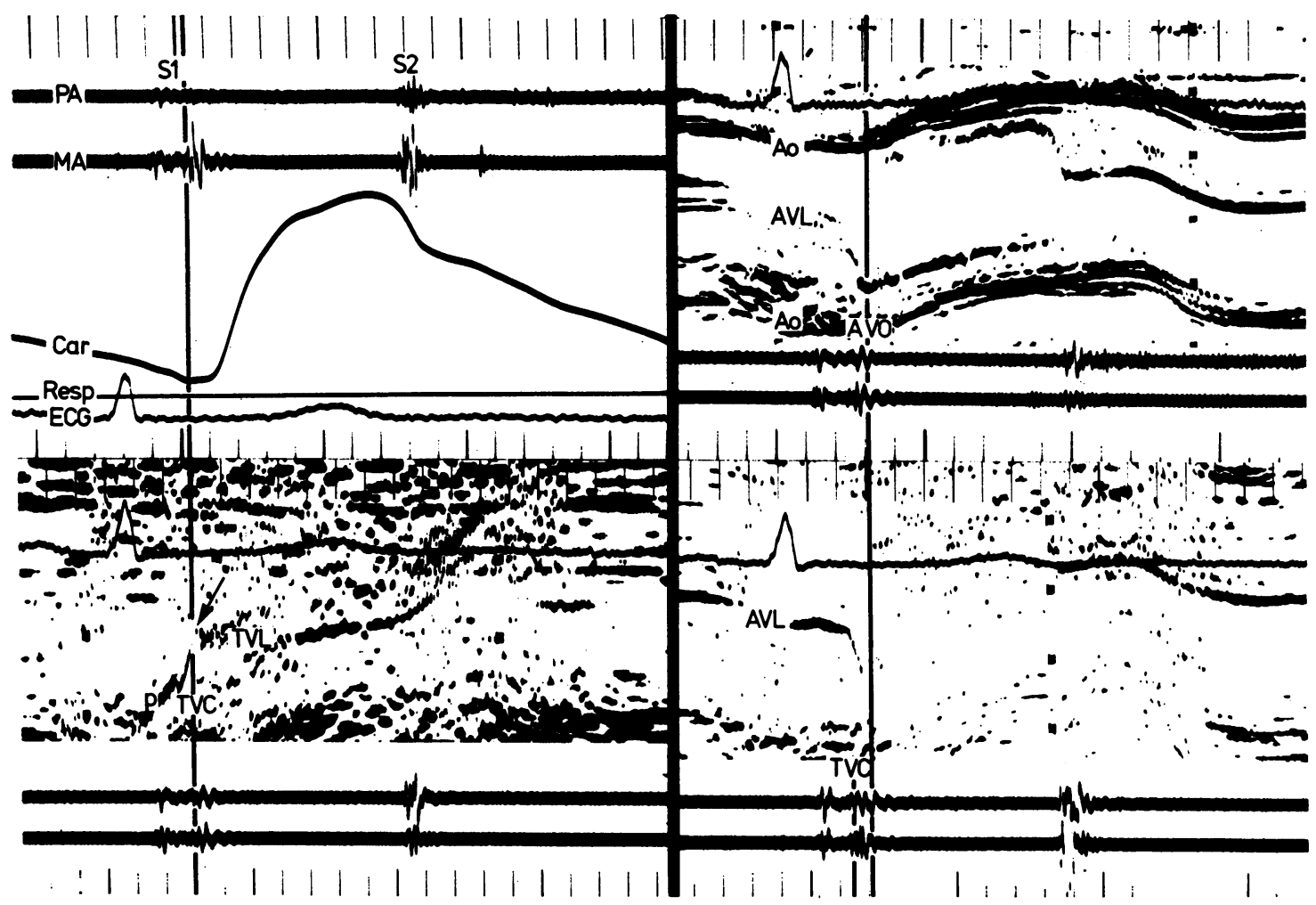

Fig. 4 Recordings from a group 3 patient mistakenly thought on auscultation to have an aortic ejection sound, arranged as in Figs 2 and 3. The phonocardiogram is very similar, except that the sound precedes the carotid upstroke by $40 \mathrm{~ms}$, and is shown to coincide with tricuspid closure (arrowed), even though there is no ECG evidence of conduction delay. The aortic valve echoes are normal, both in the conventional and apical views, and maximal valve opening does not occur until after the suspect sound.

\section{Discussion}

Our aim has been to employ the non-invasive techniques of echo- and phonocardiography to establish diagnostic criteria for a non-stenotic, bicuspid aortic valve. Aortic ejection sounds are a feature of aortic valve stenosis, but one of us (A.L.) had noted that these sounds might also be heard in patients without clinically detectable aortic valve obstruction. Epstein et al. (1965) had shown that the ejection sound in aortic stenosis is caused by halting of the upward motion of the stiffened, fused valve cusps. It seemed likely that a valve with only two cusps, which could not fold completely against the aortic wall during ejection, might behave in a similar manner, and so it was postulated that an aortic ejection sound, in the absence of aortic stenosis or regurgitation, might be a sign of an uncomplicated bicuspid valve. Patients with this sign were, therefore, followed up, and unequivocal evidence of the presence of a bicuspid valve was obtained in 6 cases (group 1).

Since a bicuspid aortic valve is a relatively benign abnormality and the patients with an isolated $\dot{\delta}$ ejection sound were asymptomatic, the use of cardiac $₹$ catheterisation and angiography to confirm the diagnosis could not be justified. However, the technique of simultaneous high speed echo- and phonocardiography (Leatham and Leech, 1975), using a recording paper speed of $200 \mathrm{~mm} / \mathrm{s}$ and $\Omega$ corresponding electronic magnification of the $N$ echocardiographic image, can identify accurately the origin of cardiac sounds.

It is important to appreciate that the timing of a transient mechanical event such as a valve tensing after opening or closing is indicated by the onset of $\stackrel{?}{\rightarrow}$ the associated sound; the quality and duration of $T$ the sound vibrations depend on the elasticity and damping of the vibrating structures, and the $\overparen{\nabla}$ appearance of the sound on the phonocardiogram is influenced by the type of electronic filtration employed in the amplifier. An analogous situation 
occurs when a hammer strikes a bell: the sound begins at the moment of impact, but the pitch of the note depends on the size of the bell, and its duration on the amount of damping present.

By recording the motions of all 4 cardiac valves, it is possible in most subjects to demonstrate a unique association between each high frequency sound and halting of the motion of one of the valves. In the case of an aortic ejection sound, the mitral and tricuspid valves have already closed and, with occasional exceptions, the pulmonary valve has already opened fully before the aortic cusps reach their maximal opening separation. There were only two instances in this study when another valve opened or closed so close to aortic opening as to make it difficult to be sure with which valve an early systolic sound was associated. It was an invariable finding that the onset of the vibrations of the ejection sound recorded in the patients with aortic stenosis was precisely coincident with the moment of final halting of the opening aortic valve cusps. These findings are similar to those of Waider and Craige (1975). Gamboa et al. (1964) investigated the timing of the ejection sound in semilunar valve stenosis. In pulmonary stenosis, they found a good relation between the severity of the stenosis and the interval from the QRS onset on the electrocardiogram to the ejection sound, but they could not show a similar relation in aortic stenosis. Using high-speed recordings, and eliminating variations in electromechanical delay by timing from the component of the first heart sound shown by echophonocardiography to be mitral closure, we have found that maximal aortic valve opening occurs significantly earlier $(P<0.005)$ in patients with a degree of stenosis, compared with normals and those with non-stenotic bicuspid valves. Though the time intervals are short, it is possible that this may provide a basis for the echophonocardiographic assessment of the severity of aortic stenosis.

Most of the group 3 patients had a soft ejection systolic murmur, this generally being the reason for their referral by the family or school doctor. In the 2 cases without a murmur, the ejection sound had been detected by one of us during a routine examination. Though the non-invasive assessment of the severity of aortic stenosis is often unreliable, we were able to show abnormal 'T-time' and ejection time indices in all the 5 patients in group 2 who had been shown by catheterisation to have valve gradients of 2.7 to $6.7 \mathrm{kPa}(20$ to $50 \mathrm{mmHg})$. Using the same criteria, all the measurements in group 3 , except one case with coarctation, were normal. Furthermore, none had electrocardiographic or echo evidence of left ventricular hypertrophy. Thus, despite the murmur, we do not believe the group 3 patients have significant aortic stenosis. (Of the 30 normal controls, 7 also had an audible systolic murmur.)

The intensity of the second heart sound is greater in patients with bicuspid valves than normals. The increased area of the large cusp, which is responsible for the ejection sound when it opens, also makes a loud noise when it tensions as it halts after closing.

The echocardiographic features of a bicuspid aortic valve have been described previously. Our findings support the observations of Nanda et al. (1974) that a bicuspid aortic valve is characterised by an eccentric closure line and multiple diastolic echoes. However, as Radford et al. (1976) emphasised, this is not invariably the case. Ten proven bicuspid valves had an 'eccentricity index' (aortic lumen diameter divided by twice the minimum diastolic closure line-aortic wall distance) greater than $1 \cdot 25$, but in 3 it was within the normal range of under 1.25. Of the 30 group 3 patients thought to have bicuspid valves, 8 had eccentricity indices under $1 \cdot 25$, and 6 of the 8 did not have multiple echoes either. Thus, conventional echo studies would fail to detect the bicuspid valve in 11 out of 43 of our cases, and the presence of an aortic ejection sound defined by echophonocardiographic studies is a more sensitive diagnostic feature.

In those cases where the closure line was eccentric it was invariably displaced anteriorly within the aortic lumen. This is surprising in view of the findings of Roberts (1970), who found considerable variation in the anatomical configuration of 59 necropsy cases of bicuspid aortic valves.

At first sight, it is paradoxical that a bicuspid valve appears on echo recordings to open wider than a normal valve. Inability of the cusps to fold back parallel to the wall of the aorta causes them to bulge outwards, increasing their separation as seen by the ultrasound beam traversing the aortic root.

It has been our practice to study Starr-Edwards aortic prostheses by placing the echo transducer over the point of maximal apical pulsation and directing the ultrasound beam up the aortic outflow tract to detect the ball motion, which is parallel to the axis of the aorta. A feature of a bicuspid aortic valve is that, unlike a normal valve, the cusps cannot fold away from the centre of the aortic lumen during ejection. This leads to the 'domed' appearance on angiograms. We thought the apical view might make it possible to detect this echocardiographically. The aortic valve was recorded successfully in 66 cases, which was 88 per cent of those in whom it was attempted. Proof that we were recording the aortic valve was provided by the identical timing of the opening and closing phases of its motion 


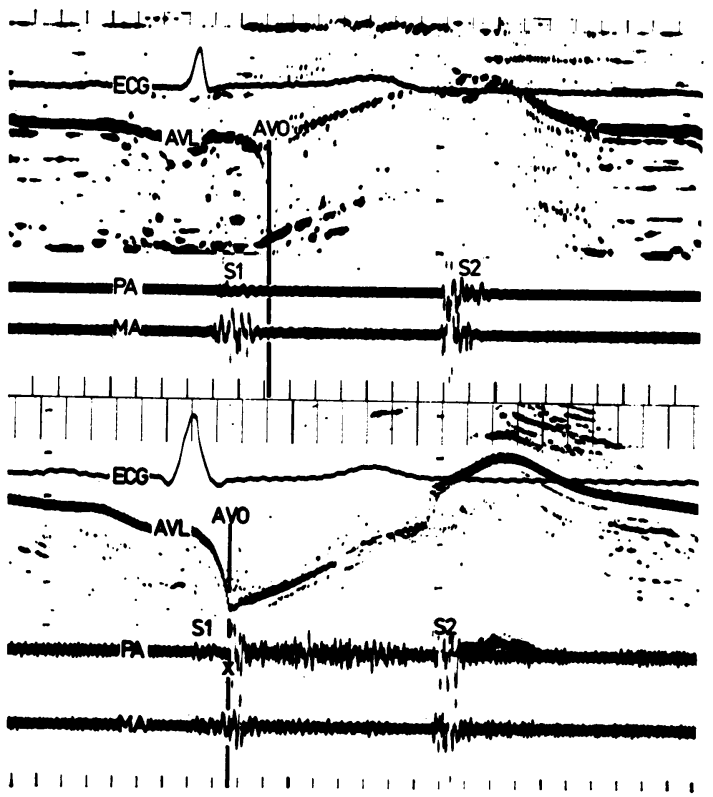

Fig. 5 Apical aortic valve echo in a normal subject (top) and a patient with a surgically demonstrated bicuspid aortic valve (bottom). Cusp echoes are seen during systole in the lower trace, and maximal valve opening coincides with the onset of the ejection sound.

when compared with the conventional view. In addition, its relation to the mitral valve and the similarity of the tracing to that of the pulmonary valve when seen from the third intercostal space (i.e. from the same relative angle) helped identification during the study.

In patients with proven bicuspid valves (group 2), echoes from the aortic valve cusps remained visible during systole. In contrast, cusp echoes were not $\stackrel{\vec{F}}{\rightarrow}$ detected during systole in the normal controls? (Fig. 5). The presence of valve echoes during $\frac{\bar{c}}{\bar{s}}$ systole suggests only that the cusps cannot fold $\vec{\sigma}$ completely parallel to the aortic wall, and this $\propto$ appearance is, therefore, also seen in congenital $\omega$ aortic valve stenosis with a tricuspid or uni- $\vec{\circ}$ cuspid valve, and in rheumatic aortic stenosis. In addition in patients with low stroke output, for $\vec{\omega}$ example in dilated cardiomyopathy or ischaemic $?$ disease, incomplete aortic valve opening sometimes? produces 'fluttering' echoes during systole. How- $\overrightarrow{0}$ ever, with these exceptions, we believe that detection 0

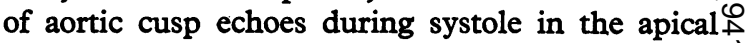
view is a sign of a bicuspid aortic valve.

An aortic ejection sound (Fig. 6) is recognised ${ }^{\circ}$ clinically as an early systolic sound, giving the $\overrightarrow{C D}$ impression of wide splitting of the first heart sound. The following features are helpful in making the $\mathbb{\mathbb { Q }}$ diagnosis on auscultation; (a) it is a loud sound and $\bar{O}$ its intensity does not vary with respiration; (b) it ㄴ. is heard over the whole praecordium, but its $\vec{c}$ relation to the first sound, and thus the impression of 'wide splitting of the first sound', is best obtained. at the apex, whereas true splitting of the first sound is most obvious at the lower left sternal edge; and (c) the aortic component of the second heart sound is usually loud.

Even so, it is possible to be mistaken. Analysis $\stackrel{\AA}{\circ}$ of our recordings leads us to conclude that 7 of the $\overrightarrow{\overrightarrow{0}}$ 37 patients originally thought to have bicuspid 3 aortic valves do not in fact have an aortic ejection sound. The commonest error was to mistake wide physiological splitting of the first sound for an aortic ejection sound. An atrial sound preceding the first sound may cause difficulties, but it is of much lower frequency and usually varies with 3 . respiration. With physiological splitting of the

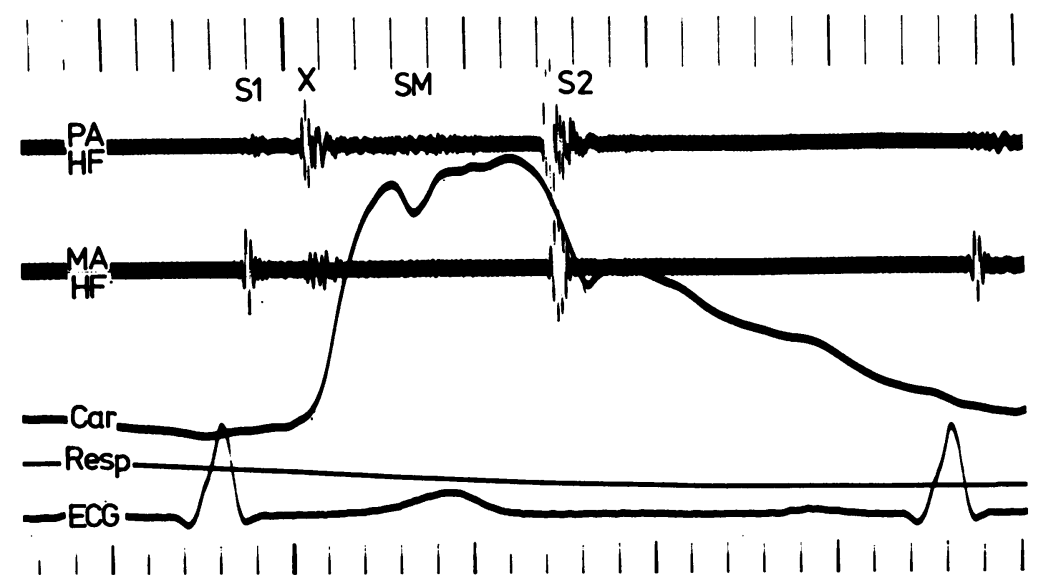

Fig. 6 Auscultatory features of an aortic ejection sound. Note the timing relative to the carotid upstroke, the soft systolic murmur and the loud second sound. 
first sound, the gap is usually narrower, but great difficulty may be encountered in right bundlebranch block, since the timing of the delayed tricuspid closure sound may be identical to an aortic ejection sound and if it is loud, as in atrial septal defect, the two may be indistinguishable.

Ejection sounds associated with pulmonary hypertension may be difficult to distinguish from aortic ejection sounds, since with raised pulmonary artery pressure ejection is delayed and the timing is similar to that of a left-sided sound (identical in the case of Eisenmenger ventricular septal defect). Unlike pulmonary stenosis, there is little variation in amplitude with respiration since atrial contraction cannot partially raise the valve in the face of high pulmonary artery end-diastolic pressure. Finally, in the case of congenitally 'floppy' mitral valves with a mid-systolic click, the click may occur sufficiently early in systole to be mistaken for an aortic ejection sound. In difficult cases, echophonocardiographic studies are invaluable in clarifying the diagnosis.

Whether an isolated aortic ejection sound can arise from an abnormality of the ascending aorta, the so-called 'root' sound, is questionable. The 'root' sound said to be found in coarctation of the aorta is, in our experience, invariably accompanied by the echo- and phonocardiographic signs of a bicuspid aortic valve, an association that is well recognised and has been said to occur in up to 75 per cent of cases of coarctation (Smith and Matthews, 1955). So far, our clinical impression of a root sound has nearly always been shown by echophonocardiographic studies to be fallacious, the suspect sound usually coinciding with late tricuspid valve closure. The only exceptions are with the enormous aorta of severe Fallot's tetralogy or pulmonary atresia and it is even possible that the ejection sounds in these patients may result from aortic valve abnormalities (Glancy et al., 1968). Thus, while not denying the possibility that such sounds may result from a greatly dilated aortic root, we share the scepticism expressed by Hancock (1961) concerning their existence.

In conclusion, we believe that the finding of an aortic ejection sound, in the absence of other signs of aortic stenosis, indicates a non-stenotic, bicuspid aortic valve. To be certain of the origin of the sound, it is necessary to record it simultaneously with echocardiograms from all 4 valves to show unique coincidence between the point of halting of the opening aortic valve cusps and the onset of the vibrations of the sound. There are several echo characteristics usually seen with bicuspid

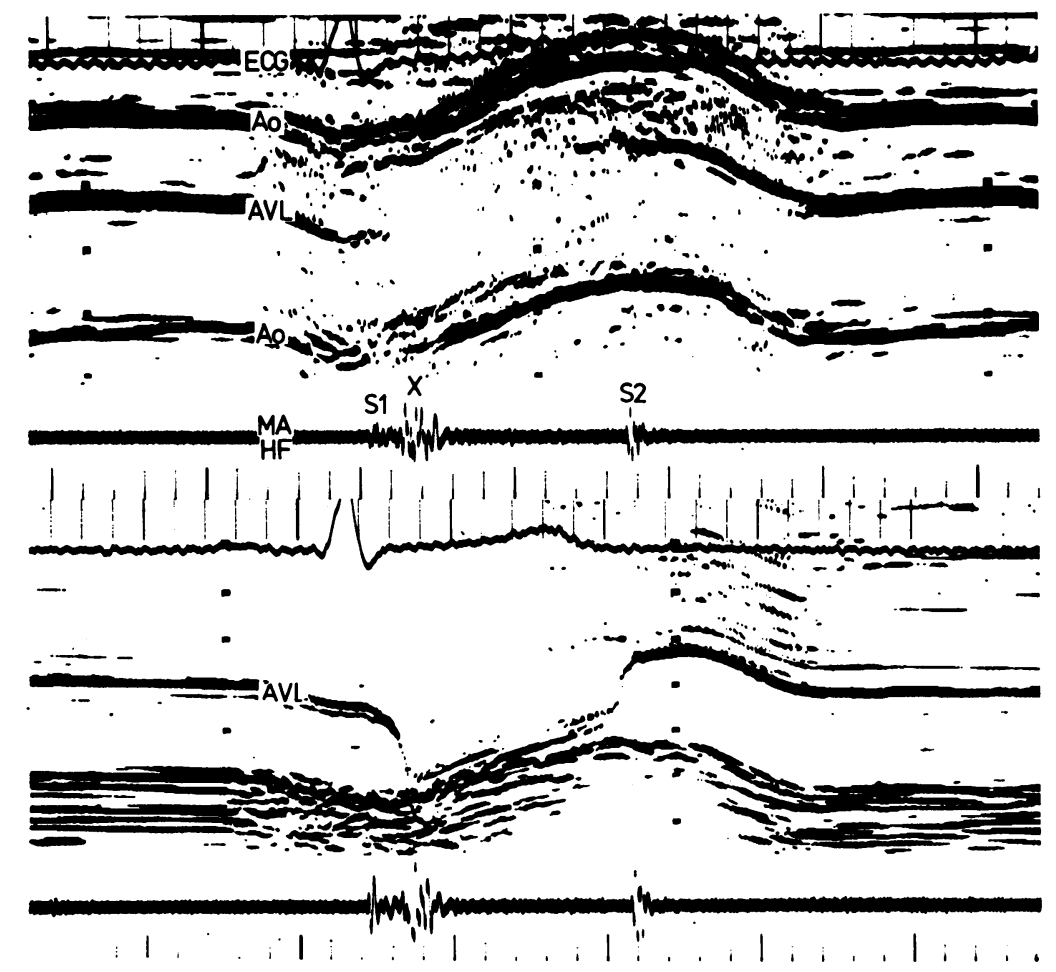

Fig. 7 Aortic valve echoes from one of the group 3 patients. Despite every effort, the cusps could not be visualised in the conventional view (top), so timing of the ejection sound could not be confirmed. However, in the apical view (bottom), the onset of the sound is seen to coincide with maximal valve opening and echoes are recorded during systole. 
valves which lend support to the diagnosis. Of particular value are the records obtained by recording the aortic valve from the apex (Fig. 7). This allows both verification of the ejection sound timing and detection of echoes from the valve during systole.

We thank Mr Mark Braimbridge and Mr Charles Drew for letting us study their surgical patients. We are particularly grateful to the British Heart Foundation, whose financial support made this work possible.

\section{References}

Epstein, E. J., and Coulshed, N. (1964). Assessment of aortic stenosis from the external carotid pulse wave. British Heart fournal, 26, 84-96.

Epstein, E. J., Criley, J. M., Raftery, E. B., Humphries, J. O., and Ross, R. S. (1965). Cineradiographic studies of the early systolic click in aortic valve stenosis. Circulation, 31, 842-853.

Gamboa, R., Hugenholtz, P., and Nadas, A. S. (1964). Accuracy of the phonocardiogram in assessing severity of aortic and pulmonic stenosis. Circulation, 30, 35-46.

Glancy, D. L., and Epstein, S. E. (1971). Differential diagnosis of type and severity of obstruction to left ventricular outflow. Progress in Cardiovascular Diseases, 14, 153-191.

Glancy, D. L., Morrow, A. C., and Roberts, W. C. (1968). Malformations of the aortic valve in patients with tetralogy of Fallot. American Heart fournal, 76, 755-759.

Hancock, E. W. (1961). Differentiation of valvar, subvalvar and supravalvar aortic stenosis. Guy's Hospital Reports, 110, 1-30.

Leatham, A., and Leech, G. J. (1975). Observations on the relation between heart sounds and valve movements by simultaneous echo- and phonocardiography (abstract). In Proceedings of the British Cardiac Society. British Heart Fournal, 37, 557 .
Leatham, A., Segal, B., and Shafter, H. (1963). Auscultatory and phonocardiographic findings in healthy children with systolic murmurs. British Heart fournal, 25, 451-459.

Leatham, A., and Vogelpoel, L. (1954). The early systolic $\overline{\bar{N}}$ sound in dilatation of the pulmonary artery. British Heart Fournal, 16, 21-33.

Lian, C., and Welti, J. J. (1937). Le claquement arteriel pulmonaire protosystolique. Archives des Maladies $d u$ Coeur et des Vaisseaux, 30, 946-954.

Nanda, N. C., Gramiak, R., Manning, J., Mahoney, E. B., Lipchik, E. O., and DeWeese, J. A. (1974). Echocardio- $\vec{\omega}$ graphic recognition of the congenital bicuspid aortic valve. Circulation, 49, 870-875.

Oakley, C. M., and Hallidie-Smith, K. A. (1967). Assessment of site and severity in congenital aortic stenosis. British Heart Fournal, 29, 367-379.

Radford, D. J., Bloom, K. R., Izukawa, T., Moes, C. A. F., and Rose, R. D. (1976). Echocardiographic assessment of bicuspid aortic valves; angiographic and pathological $O$ correlates. Circulation, 53, 80-85.

Roberts, W. (1970). The congenitally bicuspid aortic valve. American fournal of Cardiology, 26, 72-83.

Smith, D. E., and Matthews, M. B. (1955). Aortic valvular stenosis with coarctation of the aorta. British Heart fournal, 17, 198-206.

Waider, W., and Craige, E. (1975). First heart sound and ejection sounds; echocardiographic and phonocardiographic correlation with valvular events. American fournal of Cardiology, 35, 346-356.

Wauchope, G. M. (1928). The clinical importance of variations in the number of cusps forming the aortic and pulmonary valves. Quarterly fournal of Medicine, 21, 383. Weissler, A. M., Harris, W. S., and Schoenfeld, C. D. (1968). Systolic time intervals in heart failure in man. Circulation, 37, 149-159.

Wolferth, C. C., and Margolies, A. (1940). Heart sounds. In The Diagnosis and Treatment of Cardiovascular Disease, pp. 545-592. Ed. by W. C. Stroud. F. A. Davies, Philadelphia.

Requests for reprints to $\mathrm{Mr}$ Graham J. Leech, Cardiac Department, St George's Hospital, Hyde Park Corner, London SW1X 7EZ. 